\title{
The $S$-Transform and Its Inverses: Side Effects of Discretizing and Filtering
}

\author{
Carine Simon, Sergi Ventosa, Martin Schimmel, Alexander Heldring, Juan Jo Dañobeitia, Josep Gallart, and
} Antoni Mànuel

\begin{abstract}
The aim of this paper is to present a study on the potential and limits of the $S$-transform and its inverses. The $S$-transform is an extension of the short-time Fourier transform with characteristics of the wavelet transform. It is mostly used for time-frequency analyses. Two different inverse $S$-transforms have been presented in the literature. We explain why the most recent one is an approximation but a very good one. The level of approximation is calculated in this paper. We then discuss the relative merits of both inverses. A careful study enables us to show that, although both inverses are nearly exact in the infinite continuous domain, this is not true anymore in the practical finite discrete domain. Side effects are quantified, and typical examples are given. Time-frequency filtering is one of the main applications of the $S$-transform. We evaluate the effects that occur when using the $S$-transform and its inverses for filtering.
\end{abstract}

Index Terms-Local spectra, $S$-transforms, time-frequency analysis, time-frequency localization, time-varying filters.

\section{INTRODUCTION}

$\mathbf{I}$ $\mathrm{N}$ many applications such as seismology, speech processing, or astronomy, signals are not stationary, obliging to work on local spectra rather than global ones. This can be achieved with several techniques, among which one of the first has been the short-time Fourier transform (STFT) [1]. The STFT is a fixed-resolution analysis where sliding windows are used to obtain time-localized spectra. One of the drawbacks of the STFT is that it exhibits poor time resolution at high frequencies, and it cannot accurately resolve low frequencies whose periods are longer than the duration of the window. An extension of STFT, lying between it and wavelets [2], is the $S$-transform (ST) [3]. In this method, the frequency-dependent window allows for a frequency-dependent resolution with narrower windows at higher frequencies and wider windows at lower frequencies. This transform has already been used in different applications ranging from medicine [4], [5] to geophysics [6], [7].

Manuscript received September 15, 2006; revised February 26, 2007. The associate editor coordinating the review of this manuscript and approving it for publication was Dr. Zidong Wang. This work was supported by the projects SigSensual ref. REN2003-08341-C03- C01-02 and CTM2004-04510-C03-02. The work of C. Simon and S. Ventosa is financed by the I3P program. The work of M. Schimmel and J. Gallart is supported through the Ramon y Cajal and the Consolider-Ingenio 2010 Nr. CSD2006-00041 program.

C. Simon, S. Ventosa, J. J. Dañobeitia are with Unidad de Tecnología Marina, CSIC, 08012 Barcelona, Spain (e-mail: csimon@utm.csic.es).

M. Schimmel and J. Gallart are with the Institute of Earth Sciences Jaume Almera, CSIC, 08028 Barcelona, Spain.

A. Heldring is with the Department of Signal Processing and Communications, UPC, 08034 Barcelona, Spain (e-mail: heldring@tsc.upc.edu).

A. Mànuel is with the Technical University of Catalonia, UPC, 08800 Vilanova i la Geltrú, Spain.

Digital Object Identifier 10.1109/TSP.2007.897893
One of the advantages of the ST is that it is theoretically perfectly invertible [3], although the practical implementation (back transform) may lead to artifacts, when the local spectra are modified. In [8], an alternative approach to compute the inverse is proposed. The motivation for this approach was to reduce artifacts caused in filter applications when the time-frequency spectra are modified before the back transform. We show in Section III that the definition of the inverse given by [8] contains an approximation, which can, however, be made arbitrarily small. In this paper, we also analyze and compare these two inverses, in the continuous domain (Section III). As will be discussed in Section IV, important side effects can occur when discretizing both the direct ST and its inverses. Before concluding, we will study in Section V the effects of both inverse $S$-transforms (ISTs) when filtering. But first, let us briefly recall the properties of the $S$-transform in Section II.

\section{THE $S$-TRANSFORM}

The ST of a time series $u(t)$ is defined as [3]

$$
S(\tau, f)=\int_{-\infty}^{\infty} u(t) w(t-\tau, f) \mathrm{e}^{-2 i \pi f t} \mathrm{~d} t
$$

where the window $w$ needs to be 1 mean, as follows:

$$
\int_{-\infty}^{\infty} w(t, f) \mathrm{d} t=1 \quad \forall f \in \mathbb{R} .
$$

The most usual chosen window $w$ is the Gaussian one

$$
w(t, f)=\frac{|f|}{k \sqrt{2 \pi}} \mathrm{e}^{-\frac{f^{2} t^{2}}{2 k^{2}}}, \quad k>0
$$

in which $f$ is the frequency, $t$ and $\tau$ the time variables, and $k$ a scaling factor that controls the number of oscillations in the window. When $k$ increases, the frequency resolution increases, with a corresponding loss of time resolution. It should be noted that the Gaussian window has a frequency-dependent variance $\sigma^{2}=(k / f)^{2}$.

As shown in [3], (1) can be equivalently written in terms of the Fourier transform (FT) of the signal, taking advantage of the fact that the FT of a Gaussian is a Gaussian:

$$
S(\tau, f)=\int_{-\infty}^{\infty} U(\alpha+f) \mathrm{e}^{-\frac{2 \pi^{2} \alpha^{2} k^{2}}{f^{2}}} \mathrm{e}^{2 i \pi \alpha \tau} \mathrm{d} \alpha
$$




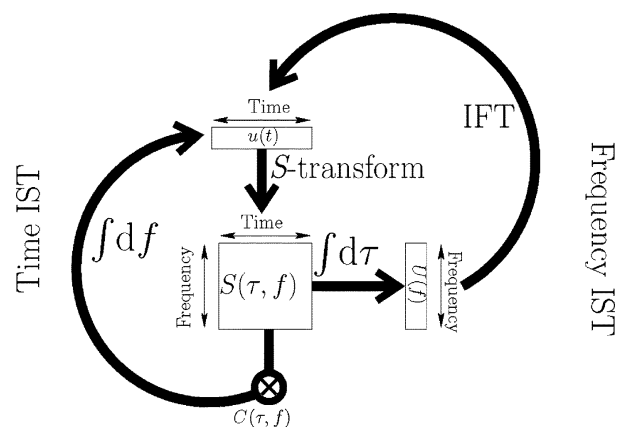

Fig. 1. Two different inverse $S$-transforms, (11) and (8). $C(\tau, f)=$ $(k \sqrt{2 \pi} /|f|) \mathrm{e}^{2 i \pi f \tau} ; \operatorname{cf}(11)$ and (10).

where $U(f)$ is the Fourier transform of $u(t)$ [see (5)]. This way of writing the ST will be called the frequency ST in the following, whereas the other way, (1), will be called the time ST.

\section{INVERSE $S$-TRANSFORM: CONTINUOUS CASE}

As the ST contains redundant information, more than one inverse can exist. As illustrated in Fig. 1, the two inverses presented here are based on different philosophies: the frequency IST (Section III-A) sums over time to yield the FT of the signal, whereas the time IST (Section III-B) sums over frequency and transforms directly back to the time domain.

\section{A. Frequency Inverse S-Transform}

We define the Fourier transform $U(f)$ of a signal $u(t)$ and its inverse as

$$
\begin{aligned}
U(f) & =\int_{-\infty}^{\infty} u(t) \mathrm{e}^{-2 i \pi f t} \mathrm{~d} t \\
u(t) & =\int_{-\infty}^{\infty} U(f) \mathrm{e}^{2 i \pi f t} \mathrm{~d} f .
\end{aligned}
$$

As observed in [3], one of the nice properties of the ST is that it is perfectly invertible. Indeed by summing over all times and using (2), we obtain the FT of the original signal

$$
\int_{-\infty}^{\infty} S(\tau, f) \mathrm{d} \tau=U(f)
$$

where $U(f)$ is the FT of $u(t)$.

Therefore, it is easy to go back to the original signal by summing over all frequencies. Finally, we obtain

$$
\iint_{-\infty}^{\infty} S(\tau, f) \mathrm{e}^{2 i \pi f t} \mathrm{~d} \tau \mathrm{d} f=u(t)
$$

\section{B. Time Inverse S-Transform}

Following [8], we start by defining the following set of time-time functions:

$$
x_{f}(\tau, t)=u(t) \mathrm{e}^{-\frac{f^{2}(\tau-t)^{2}}{2 k^{2}}} .
$$

It is obvious that $\forall f, x_{f}(t, t)=u(t)$. Now, from $x_{f}$, it is possible to go back to the ST up to some constant factor

$$
X(\tau, f)=\int_{-\infty}^{\infty} x_{f}(\tau, t) \mathrm{e}^{-2 i \pi f t} \mathrm{~d} t=\frac{k \sqrt{2 \pi}}{|f|} S(\tau, f) .
$$

It is important to notice that contrarily to what is written in [8], this is not an FT due to the dependence of $x_{f}$ on $f$. However, we will show here that this inverse, although not completely exact, is a very good approximation.

Based on (10), in [8], the following inverse is proposed:

$$
\tilde{u}(\tau)=\int_{-\infty}^{\infty} X(\tau, f) \mathrm{e}^{2 i \pi f \tau} \mathrm{d} f .
$$

This equation can be rewritten in the following way:

$$
\begin{aligned}
\tilde{u}(\tau) & =\iint u(t) \mathrm{e}^{-\frac{f^{2}(\tau-t)^{2}}{2 k^{2}}} \mathrm{e}^{2 i \pi f(\tau-t)} \mathrm{d} f \mathrm{~d} t \\
& =\int_{-\infty}^{\infty} u(t)\left(\int_{-\infty}^{\infty} \mathrm{e}^{-\frac{f^{2}(\tau-t)^{2}}{2 k^{2}}} \mathrm{e}^{2 i \pi f(\tau-t)} \mathrm{d} f\right) \mathrm{d} t .
\end{aligned}
$$

If we set

$$
I(x)=\int_{-\infty}^{\infty} \mathrm{e}^{-\frac{f^{2} x^{2}}{2 k^{2}}} \mathrm{e}^{2 i \pi f x} \mathrm{~d} f
$$

we have

$$
\tilde{u}(\tau)=u * I(\tau)
$$

For the inverse to be exact, $I(\tau)$ should be equal to the Dirac function. In the following, we calculate its value.

We can rewrite (14) as

$I(x)=\int_{-\infty}^{\infty} \mathrm{e}^{-\frac{f^{2} x^{2}}{2 k^{2}}} \cos (2 \pi f x) \mathrm{d} f+i \int_{-\infty}^{\infty} \mathrm{e}^{-\frac{f^{2} x^{2}}{2 k^{2}}} \sin (2 \pi f x) \mathrm{d} f$

where $i=\sqrt{-1}$. Let us set $I_{R}(x)$ the real part of $I(x)$ and $I_{I}(x)$ its imaginary part. As $\mathrm{e}^{-\left(f^{2} x^{2} / 2 k^{2}\right)}$ is even and $\sin (2 \pi f x)$ is odd, we immediately obtain $I_{I}(x)=0$. On the other hand, $\cos (2 \pi f x)$ is even, so we get

$$
I(x)=I_{R}(x)=2 \int_{0}^{\infty} \mathrm{e}^{-\frac{f^{2} x^{2}}{2 k^{2}}} \cos (2 \pi f x) \mathrm{d} f .
$$

It can be shown [9] that

$$
I(x)=\underbrace{\sqrt{2 \pi} k \mathrm{e}^{-2 \pi^{2} k^{2}}}_{C(k)} \frac{1}{|x|} \quad \forall x \in \mathbb{R} .
$$

Let us set

$$
I_{\epsilon}(x)=\epsilon|x|^{\epsilon-1}=\frac{\epsilon}{|x|}|x|^{\epsilon} .
$$


We know [10] that $\lim _{\epsilon \rightarrow 0} I_{\epsilon}(x)=\delta(x)$. Let us set $C(k)=\epsilon$ in (18). For $C(k)$ small enough, we can thus approximate $I(x)$ by $I_{\epsilon}(x)$ as $|x|^{\epsilon} \simeq 1$ for $\epsilon$ small. Therefore

$$
I(x) \simeq \delta(x)
$$

for large $C(k)$.

In reality, the constant term $C(k)$ of $I(x)(18)$ decreases rapidly with $k$. For example, $C(0.5) \simeq 0.9 \times 10^{-3}$, $C(1) \simeq 10^{-8}$ and $C(1.5) \simeq 10^{-19}$. Therefore, for $k \geq 1$, $C(k) \simeq 0$, and $I(x) \simeq \delta(x)$, where $\delta$ is the Dirac function. We thus have

$$
\tilde{u}(\tau)=u * I(\tau) \simeq u * \delta(\tau)=u(\tau)
$$

\section{COMPUTING THE $S$-TRANSFORM AND ITS INVERSES IN THE DISCRETE CASE}

In this section, we will discuss the artifacts that occur when the ST and its inverses are discretized.

\section{A. Discrete S-Transform}

Let $u[n]=u(n T), n=0, \ldots, N-1$ denote the discrete time series, corresponding to $u(t)$, with time sampling interval of $T$. Let $f_{s}=1 / T$ be the sampling frequency and $f_{0}$ be the frequency step, and $M=f_{s} / f_{0}$ and $m=-M / 2, \ldots, M / 2-1$ is the index of frequency range. We can then write the discrete ST as

$$
S[p, m]=\sum_{n=0}^{N-1} u[n] \frac{|m|}{k M \sqrt{2 \pi}} \mathrm{e}^{-\frac{1}{2}\left(\frac{m(p-n)}{M k}\right)^{2}} \mathrm{e}^{-2 i \pi \frac{n m}{M}}
$$

with $p=0, \ldots, N-1$ being the time index. In [3], the definition of the ST in the frequency domain (4) is used to compute the ST in the discrete case. It is thus computed as

$$
S[p, m]=\sum_{q=-M / 2}^{M / 2-1} U\left[\frac{m+q}{M}\right] \mathrm{e}^{-2\left(\frac{\pi q k}{m}\right)^{2}} \mathrm{e}^{2 i \pi \frac{q p}{M}}
$$

where $U[m / M]$ is the discrete FT of $u[n]$. However, great care should be taken as, while it is true that the continuous FT of a Gaussian is a Gaussian, this is not necessarily true anymore in the discrete case. In fact, to be exact, it would be necessary to compute the discrete FT of the desired Gaussian functions and not to use directly discrete Gaussians in the frequency domain. This can be understood in the following way: the flatter the Gaussian is (i.e., the bigger the standard deviation is), the more information will be lost as only a finite number of samples will be used to calculate the Fourier transform. Here, as seen in Section III, the standard deviation of the Gaussian is $\sigma=|k / f|$. Therefore, the loss on the FT of the Gaussian will differ depending on the frequency. Fig. 2 illustrates this effect. It represents the absolute value of the time [Fig. 2(b)] and frequency [Fig. 2(a)] ST of a Delta function on the frequency axis, using equations (22) and (23), respectively. We see that using (23) leads to problems at low frequencies as explained above.
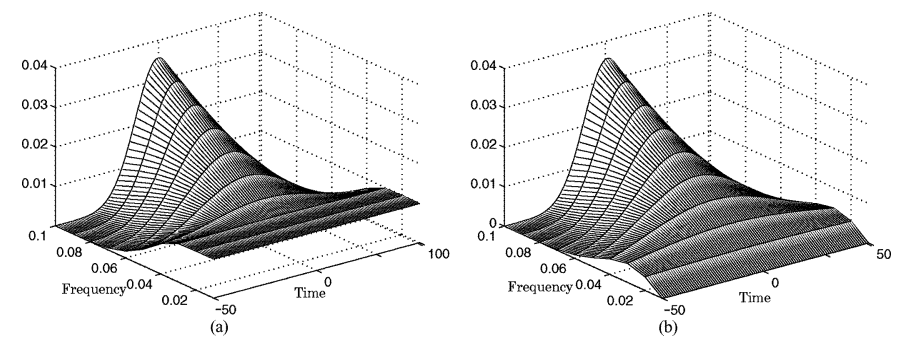

Fig. 2. Comparison between the two ways of computing the ST. (a) Absolute value of the ST of a Delta computed in the frequency domain; (b) absolute value of the ST of a Delta computed in the frequency domain.

Only low frequencies are presented in Fig. 2 as, at high frequencies, results are equivalent for both inverses.

\section{B. The Discretized Version of the Frequency Inverse $S$-Transform}

In its discrete form, we define the following inverse discrete FT:

$$
u[n]=\frac{1}{M} \sum_{m=-M / 2}^{M / 2-1} U[m] \mathrm{e}^{\frac{2 i \pi m n}{M}}
$$

for any $u$ and where $U$ is the FT of $u$.

In order to compute the inverse ST, [3] uses the formula of the ST in the frequency domain (4). They then obtain

$$
\hat{U}[m]=\sum_{p=0}^{N-1} S[p, m]
$$

where $\hat{U}$ is the estimate of $U$ and

$$
\hat{u}[n]=\frac{1}{M} \sum_{m=-M / 2}^{M / 2-1} \sum_{p=0}^{N-1} S[p, m] \mathrm{e}^{\frac{2 i \pi m n}{M}} .
$$

In order to compute (26), we need (4). But, in the previous section, we have explained that (4) is only valid in the continuous domain as in the discrete case, it leads to some side effects seen in Fig. 2.

Furthermore, because of the use of the FT, as many frequency samples as time samples $(N=M)$ are required. In the case of geophysics applications for example, this is a major drawback as we have to deal with a long time series whereas only a few frequencies are relevant. Consequently, many redundant frequency samples are calculated, leading to an inefficient algorithm.

\section{Discretized Version of the Time Inverse}

In the discrete case, $\tilde{u}(t)(11)$ can be rewritten as

$$
\begin{aligned}
\tilde{u}[n]= & \sum_{p=0}^{N-1} u[p] \frac{1}{M} \sum_{m=-M / 2}^{M / 2-1} \mathrm{e}^{-\frac{1}{2}\left(\frac{m(p-n)}{M k}\right)^{2}} \\
& \times \mathrm{e}^{-2 i \pi \frac{m}{M}(p-n)} \\
= & u \circledast I_{d}[n]
\end{aligned}
$$


where $\circledast$ is the circular convolution and

$$
I_{d}[n]=\frac{1}{M} \sum_{m=-M / 2}^{M / 2-1} \mathrm{e}^{-\frac{1}{2}\left(\frac{m n}{M k}\right)^{2}} \mathrm{e}^{2 i \pi \frac{m}{M} n} .
$$

In the continuous case, we have shown that $I(14)$, the equivalent of $I_{d}$, is nearly a Dirac function. In the discrete case, $I_{d}$ is an approximation of the Kronecker Delta function, the approximation coming from the one of $I$ and from the fact that there is a finite number of samples. The creation of artifacts is discussed in the following.

We can rewrite (28) as

$$
\tilde{u}[n]=u[n] I_{d}[0]+\sum_{p=0, p \neq n}^{N-1} u[p] I_{d}[n-p]
$$

and $I_{d}[0]=(1 / M) \sum_{m=-M / 2}^{M / 2-1} 1=1$ so the error is $\sum_{p=0, p \neq n}^{N-1} u[p] I_{d}[n-p]$. But $I_{d}[n]$ has an upper limit of

$$
\begin{aligned}
\left|I_{d}[n]\right| & \leq \frac{1}{M} \sum_{m=-M / 2}^{M / 2-1}\left|\mathrm{e}^{-\frac{1}{2}\left(\frac{m n}{M k}\right)^{2}}\right|\left|\mathrm{e}^{2 i \pi \frac{m}{M} n}\right| \\
& \leq \frac{1}{M} \sum_{m=-M / 2}^{M / 2-1} \mathrm{e}^{-\frac{1}{2}\left(\frac{m n}{M k}\right)^{2}} .
\end{aligned}
$$

Equation (31) is a sum of Gaussians with standard deviation of $|M k / n|$. As it is a sum of positive numbers, we get

$$
I_{d}[n] \leq \frac{k \sqrt{2 \pi}}{n} \quad \forall n \in \mathbb{N}^{*}
$$

where the $\mathbb{N}^{*}$ stands for the set of positive integers. Going back to (30), we see that $I_{d}[n]$ has local maxima when $\mathrm{e}^{2 i \pi(m / M) n}=$ 1 i.e., when $m n / M \in \mathbb{N}$ or when $n=a M, a \in \mathbb{N}$ as $m \in \mathbb{N}$. We easily see that the artifacts are small but with peaks at each $n=a M$ being smaller with $a$ increasing. The relation between the first maximum and the second one is

$$
\frac{I_{d}[0]}{I_{d}[M]} \geq \frac{M}{k \sqrt{2 \pi}} .
$$

As in the continuous case, the precision of the method depends on the choice of $k$ but, in the discrete case, we also have to take into account the sample numbers. Equation (33) is an important feature since it helps for the choice of $M$ and $k$ for each application.

In order to have a better idea of the function $I_{d}$, we have plotted it for $M=101$ and for $k=0.5$ to 5 in Fig. 3. It shows a zoom on the horizontal and vertical axis for visual purposes. Note that the amplitude of the middle sample is 1 for all values of $k$ and that the curves are very similar outside the window shown in Fig. 3. We can see how close it is to a Kronecker Delta. Only $k=0.5$ to 2.5 are pointed out as the other ones are too close to a Delta to be really distinguishable. As explained in Section III-B for the continuous case, for $k \geq 1, I_{d}$ (or $I$ ) is a very good approximation of a Delta (or Dirac) function.

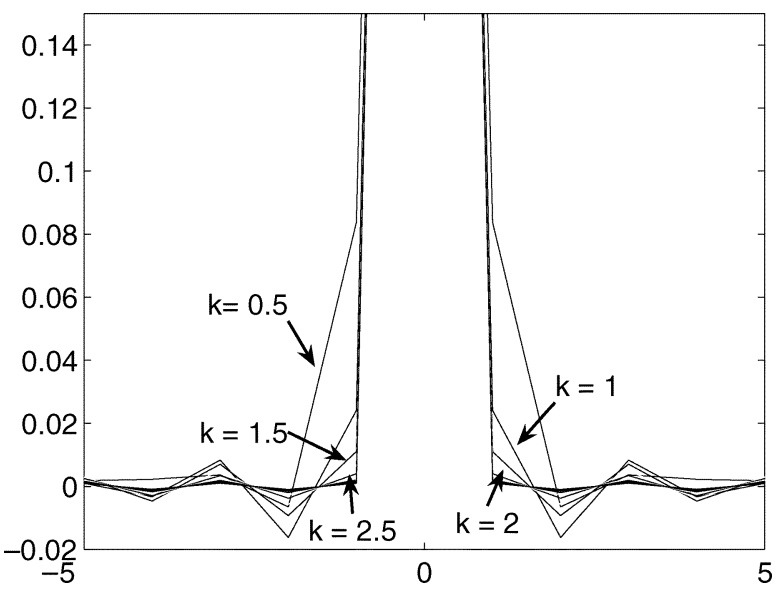

Fig. 3. Effect of the discretization of the time inverse ST: $I_{d}$ for $M=101$ points with $k=0.5$ to 5 (horizontal zoom between -5 and 5 , vertical zoom was applied as all curves behave as a Delta function at the middle sample). If perfect, $I_{d}$ should be equal to a Delta.

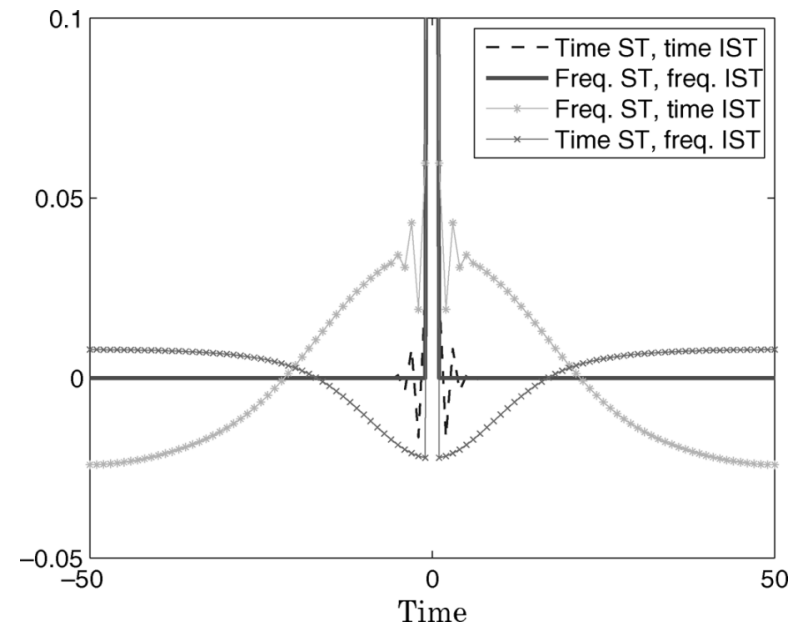

Fig. 4. Time-domain Delta function, transformed to time-frequency domain and back to time domain using the four possible combinations of ST-IST pairs mentioned in this paper.

\section{Comparing the Side Effects Between Both Inverses}

In order to further illustrate the problem of the side effects, we will show the simple case of a Delta function in Fig. 4. We will compute its ST both time (22) and frequencywise (23). We then use both inverses, (26) and (27). If perfect, the result should thus be the original Delta function. In the plot, "freq. ST" corresponds to (23), "time ST" to (22), "freq. inverse" to (26), and "time inverse" to (27). Interesting conclusions can be drawn from this figure: the side effects of the frequency ST and of the frequency inverse nearly cancel out when the signal is not modified.

Furthermore, the artifacts of the frequency ST sum with those of the time IST as does the time ST with the frequency IST. The last combination, i.e., time ST with time IST, leads to the small side effects already studied in Fig. 3 (Section IV-C).

We thus see that the choice of which ST and which inverse, although equivalent in the continuous infinite domain, is far from being neutral in the discrete finite case. Out of Fig. 4, it seems to be better not to mix frequency and time method, i.e., either the frequency ST and frequency inverse should be applied or the 

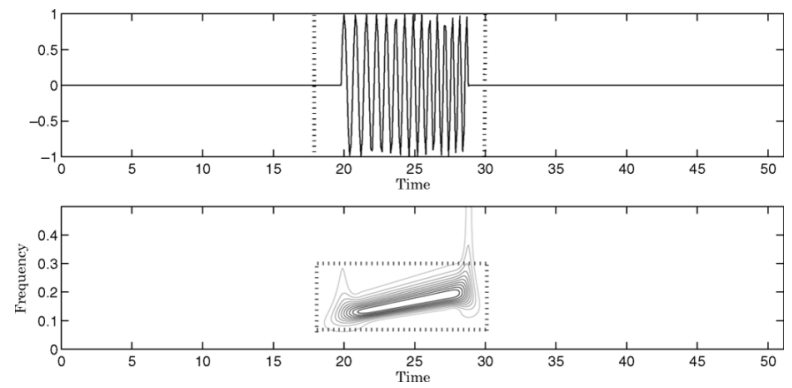

Fig. 5. Original chirp used in [8, Fig. 2] and its ST. Inside the dotted lines, a 0 -weight will be applied. Outside the dotted lines, a 1-weight will be applied.

time ST and time inverse. Obviously, none of the combinations completely avoid all the artifacts.

In order to have an insight on the practical implications of these effects, the example of Fig. 2 of [8] is taken: a limited time chirp is used as the basic signal (see the top graph of Fig. 5). Its time ST is plotted in the bottom graph of Fig. 5, with the dotted lines representing the mask that will be applied on it. The mask equals 1 everywhere except inside the rectangle where it is null. Therefore, only the part inside the rectangle should be removed.

The side effects that occur when filtering are somewhat different to [8] and shown in Fig. 6(a) and (b). More details on the consequences of the use of the ST when filtering are detailed in the following section. This example clearly shows the different consequences of the discretization when using the time or frequency ST and its inverses. The lack of time localization is clearly seen on both top plots where oscillations occur outside of the limits of the chirp. Furthermore, we see the importance of using the time ST when using the time IST.

Note that in parallel and independently to this contribution, [11] has studied the error made when using the discrete-time IST. However, he used the discrete-frequency ST in combination with the discrete-time IST, introducing some low-frequency error as shown in the bottom plot of Fig. 6(a). The frequency errors in Figs. 1(c) and 2(c) of [11] could have been avoided by using the time ST-IST combination.

\section{FILTERING With $S$-TRANSFORM}

One of the interests of the ST lies in the capacity of filtering a signal in the time-frequency domain before going back to the time-filtered signal. After filtering with a filter $F(t, f)$, we are led to the following signals:

$$
\begin{aligned}
& u_{F 1}(t)=\iint_{-\infty}^{\infty} S(\tau, f) F(\tau, f) \mathrm{e}^{2 i \pi f t} \mathrm{~d} \tau \mathrm{d} f \\
& u_{F 2}(t)=k \sqrt{2 \pi} \int_{-\infty}^{\infty} \frac{S(t, f) F(t, f)}{|f|} \mathrm{e}^{2 i \pi f t} \mathrm{~d} f
\end{aligned}
$$

where $u_{F 1}$ and $u_{F 2}$ are the filtered versions of $u$ using the frequency (8) and time (11) inverses, respectively. In the following, we are going to study the correctness of using ST for filtering. In order to get some insights on the effects, we will study two specific cases: a time filter and a frequency filter. Here, we define the time and frequency filters as bandpass, which act either on time or frequency without affecting the other variable. It should be noticed that the side effects shown in the next two sections would be the same if a separable time-frequency filter $F(t, f)$ were applied, i.e., if it could be written as $F(t, f)=F_{1}(t) F_{2}(f)$.

\section{A. Time Filtering}

In this section, we consider a filter that is only time dependent. Therefore, the ST is in reality not necessary to filter the signal, and we expect the following result:

$$
u_{F}(t)=u(t) F(t) .
$$

The FT of $u_{F}$ is of the form

$$
\begin{aligned}
U_{F}(f) & =U(f) * \hat{F}(f) \\
& =\int_{-\infty}^{\infty} U(f-\alpha) \hat{F}(\alpha) \mathrm{d} \alpha
\end{aligned}
$$

where $\hat{F}$ is the FT of the filter $F$.

1) Using the Frequency Inverse: As this IST computes the FT of the signal first, we will directly study the effect of filtering on the FT of the signal.

$$
\begin{aligned}
U_{F 1}(f) & =\int_{-\infty}^{\infty} S(\tau, f) F(\tau) \mathrm{d} \tau \\
& =\iint_{-\infty}^{\infty} U(f+\alpha) \mathrm{e}^{-\frac{2 \pi^{2} \alpha^{2}}{k^{2} f^{2}}} \mathrm{e}^{2 i \pi \alpha \tau} F(\tau) \mathrm{d} \tau \mathrm{d} \alpha \\
& =\int_{-\infty}^{\infty} U(f-\alpha) \mathrm{e}^{-\frac{2 \pi^{2} \alpha^{2}}{k^{2} f^{2}}} \int_{-\infty}^{\infty} \mathrm{e}^{-2 i \pi \alpha \tau} F(\tau) \mathrm{d} \tau \mathrm{d} \alpha \\
& =\int_{-\infty}^{\infty} U(f-\alpha) \mathrm{e}^{-\frac{2 \pi^{2} \alpha^{2}}{k^{2} f^{2}}} \hat{F}(\alpha) \mathrm{d} \alpha
\end{aligned}
$$

where we made a change of variable from $\alpha$ to $-\alpha$ to go from (40) to (41).

We thus see that, instead of the expected result (38), the FT of the signal is weighted at each frequency by a Gaussian-type function $\mathrm{e}^{-\left(2 k^{2} \pi^{2} \alpha^{2} / f^{2}\right)}$. Note that due to the dependence on $\alpha / f$ of the Gaussian, $U_{F 1}(f)$ cannot be written as a convolution anymore. First, independently of the chosen signal, the effect of filtering is illustrated in Fig. 7 where a simple square box filter is chosen. The figure represents the FT of the filter with a solid line while the dashed-dotted lines are the FT of the filter weighted by the Gaussian at different frequencies: $\mathrm{e}^{-\left(2 k^{2} \pi^{2} \alpha^{2} / f^{2}\right)} \hat{F}(\alpha)$ as shown in (42). It can be seen that the filter changes a lot with the frequency and that it always loses some oscillations. Figs. 8 and 9 illustrate this effect on a couple of examples. Fig. 8 represents a simple Delta that has been filtered by a square box ranging from -15 to 15 , whereas Fig. 9 is a chirp on which the same filter has been applied. On both of them, the top part represents the time version of the filtered signal while the bottom 

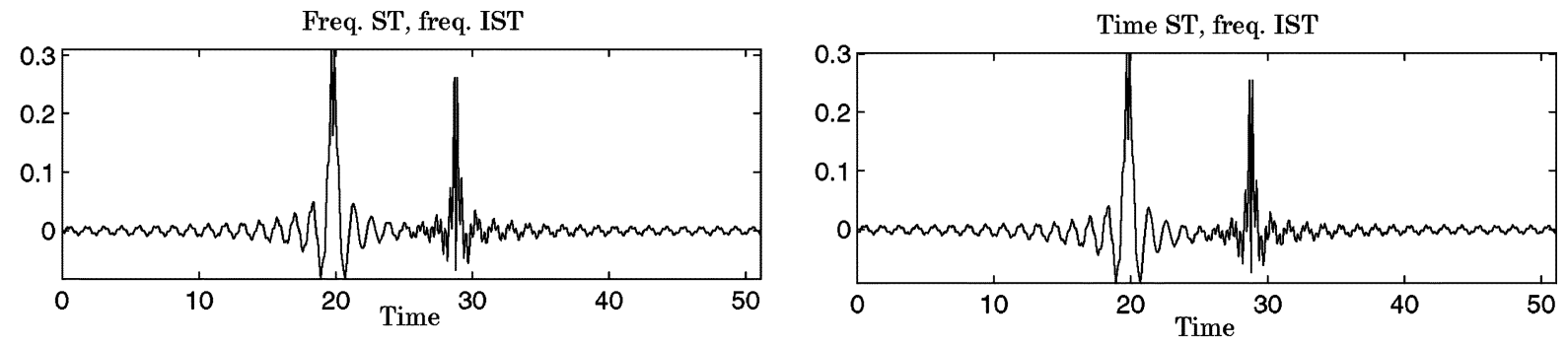

Freq. ST, time IST

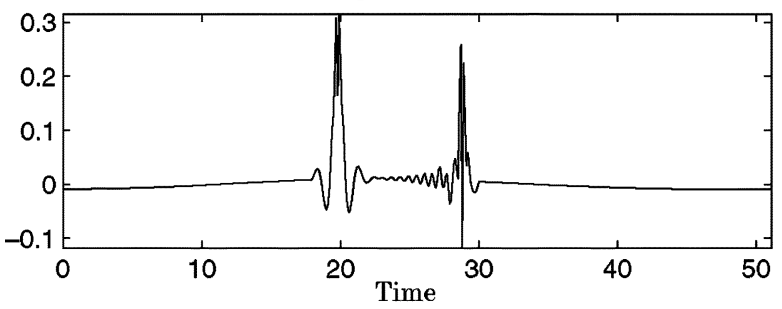

(a)

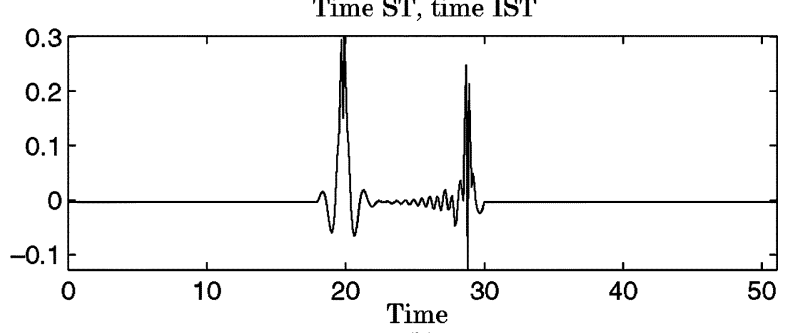

(b)

Fig. 6. Comparison of the use of the two ST-IST's procedures on the time-limited chirp Fig. 5. (a) Filtered chirp used in [8, Fig. 2]. Top plot uses a frequency ST and a frequency IST. Bottom plot uses a frequency ST and a time IST. (b) Filtered chirp used in [8, Fig. 2]. Top plot uses a time ST and a frequency IST. Bottom plot uses a time ST and a time IST.

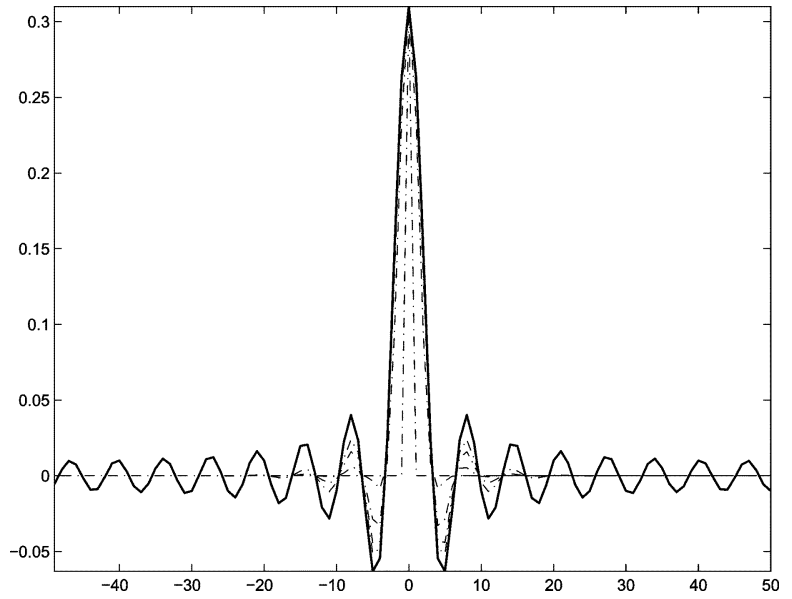

Fig. 7. Simple rectangular filter was chosen for this example. The solid line represents the FT of the filter. The dashed-dotted lines are the FT of the filter weighted by different Gaussian functions depending on the output frequency. They represent the filters used in the frequency IST instead of the desired one.

one represents its FT. On the top part of Fig. 8, a zoom has been applied for the sake of clarity.

In time domain, using Parseval's theorem, we can rewrite the filtered signal (42) in the following way:

$$
\begin{aligned}
u_{F 1}(t) & =\int_{-\infty}^{\infty} \int_{-\infty}^{\infty} u(\tau) F(\tau) \frac{|f|}{k \sqrt{2 \pi}} \mathrm{e}^{-\frac{(t-\tau)^{2} f^{2}}{2 k^{2}}} \mathrm{e}^{2 i \pi f(\tau-t)} \mathrm{d} f \mathrm{~d} \tau \\
& =u_{F}(t) * h(t)
\end{aligned}
$$

where

$$
h(t)=\frac{1}{k \sqrt{2 \pi}} \int_{-\infty}^{\infty}|f| \mathrm{e}^{-\frac{t^{2} f^{2}}{2 k^{2}}} \mathrm{e}^{2 i \pi f t} \mathrm{~d} f .
$$

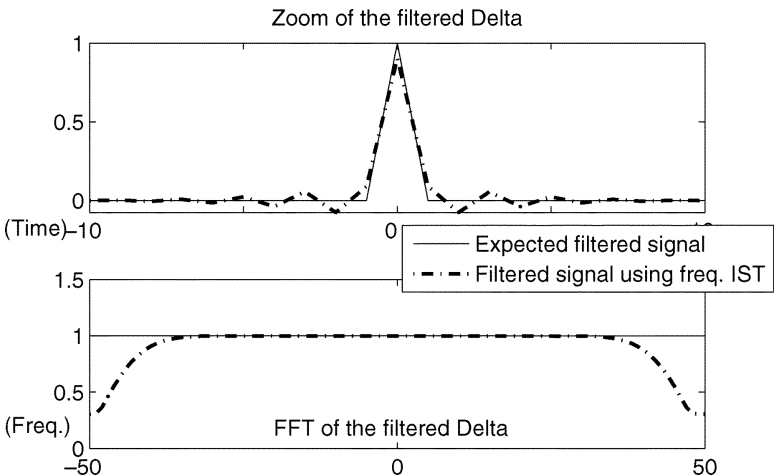

Fig. 8. Filtering of a Delta: expected result (solid line) and result through frequency IST (dashed-dotted line). The top plots represent a zoom of the filtered signal in the time domain. The bottom plots represent the filtered signal in the frequency domain.

We can show that the integral of $h(t)$ can be calculated and gives

$$
h(t)=C \frac{1}{t^{2}}
$$

where $C$ is a constant subject to

$$
C=k\left(\sqrt{2 / \pi}-2 k \pi \mathrm{e}^{-2 \pi^{2} k^{2}} \operatorname{erfi}(\sqrt{2} k \pi)\right) .
$$

When $k=1$ for example, $C \simeq-2.2 * 10^{-2}$. The filtered signal is thus not only multiplied by the time filter $F(t)$ as expected but also convoluted by $C / t^{2}$ where the value of $h(t)$ is independent of $F(t)$. This means that there is an extra windowing operation that affects the expected filtered output $u_{F}(t)$. Although $h(t)$ is well localized due to its $1 / t^{2}$ dependence, the final filter output is smeared by the convolution with $h(t)$. 


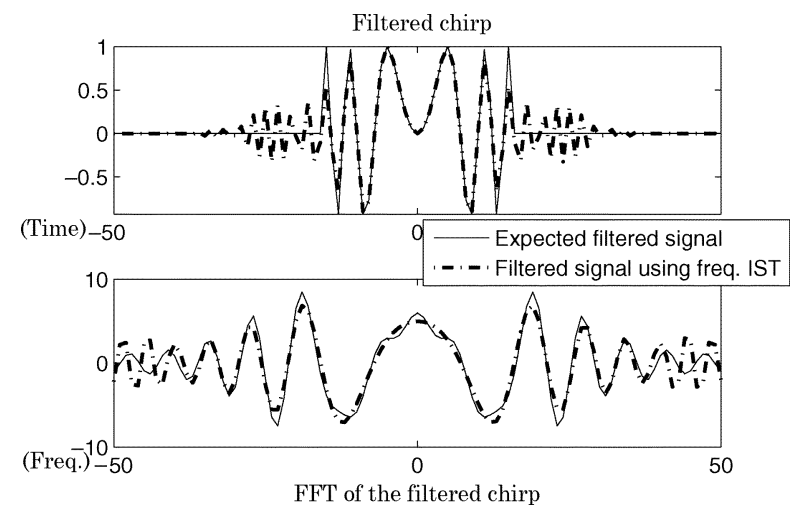

Fig. 9. Filtering of a chirp: expected result (solid line) and result through frequency IST (dashed-dotted line). The top plots represent the filtered signal in the time domain. The bottom plots represent the filtered signal in the frequency domain.

2) Using the Time Inverse: Applying (35) with $F(t, f)=$ $F(t)$ gives

$$
\begin{aligned}
u_{F 2}(t) & =k \sqrt{2 \pi} \int_{-\infty}^{\infty} \frac{S(t, f) F(t)}{|f|} \mathrm{e}^{2 i \pi f t} \mathrm{~d} f \\
& =\tilde{u}(t) F(t) \simeq u(t) F(t)
\end{aligned}
$$

which is the expected result.

\section{B. Frequency Filtering}

When a filter $F$ is only frequency dependent, it could be directly applied on the Fourier transform signal without using the ST. Therefore, the expected results are the following ones:

$$
\begin{aligned}
U_{F}(f) & =U(f) F(f) \\
u_{F}(t) & =u(t) * \check{F}(t)
\end{aligned}
$$

where $\check{F}$ is the inverse FT of the filter $F$.

1) Using the Frequency Inverse: Applying (34) with $F(t, f)=F(f)$, we get

$$
\begin{aligned}
U_{F 1}(f) & =\int_{-\infty}^{\infty} S(\tau, f) F(f) \mathrm{d} \tau \\
& =U(f) F(f)
\end{aligned}
$$

which is the expected result.

2) Using the Time Inverse:

$$
\begin{aligned}
u_{F 2}(t) & =k \sqrt{2 \pi} \int_{-\infty}^{\infty} \frac{S(t, f) F(f)}{|f|} \mathrm{e}^{2 i \pi f t} \mathrm{~d} f \\
& =u * I_{F}(t)
\end{aligned}
$$

where, by analogy with (11), we have defined $I_{F}(t)=$ $\int_{-\infty}^{\infty} \mathrm{e}^{-\left(f^{2} t^{2} / 2 k^{2}\right)} \mathrm{e}^{2 i \pi f t} F(f) \mathrm{d} f$.

If we compare (54) with the time-domain filtering (50), we see that the inverse FT of the filter $\breve{F}(t)=\int_{-\infty}^{\infty} F(f) \mathrm{e}^{2 i \pi f t} \mathrm{~d} f$ is weighted by a time-frequency-dependent Gaussian

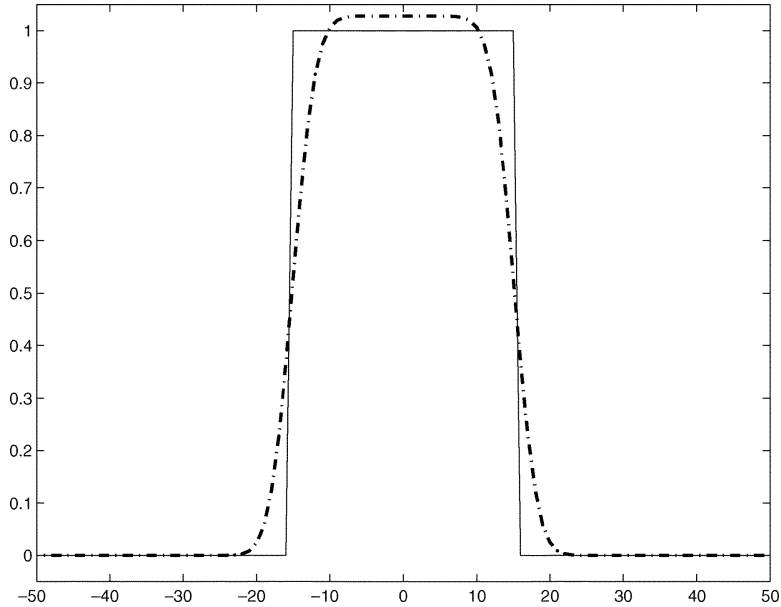

Fig. 10. Simple rectangular filter in the frequency domain was chosen for this example. The solid line represents this filter. When using the time IST (11), instead of a product of the FT of the original signal by this filter, the FT of the signal is multiplied by the filter shown in dashed-dotted line.

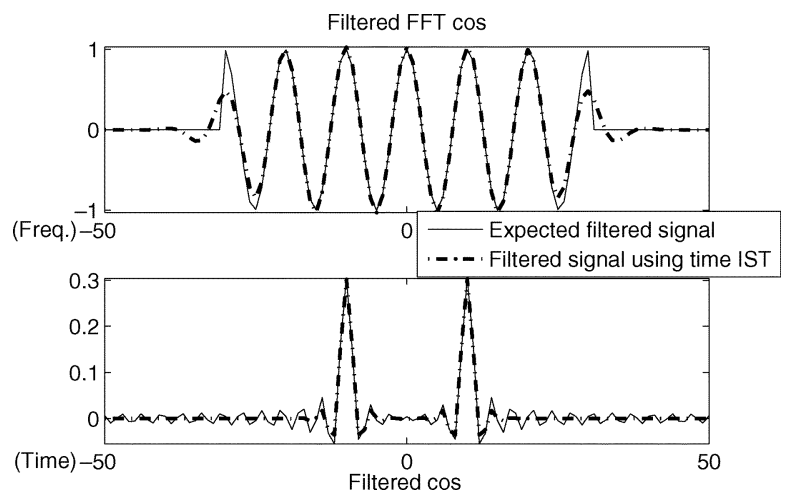

Fig. 11. Filtering of an IFT of a cos: expected result (solid line) and result through time IST (dashed-dotted line). The top plots represent the filtered signal in the frequency domain. The bottom plots represent the filtered signal in the time domain.

$\mathrm{e}^{-\left(f^{2} t^{2} / 2 k^{2}\right)}$. We first illustrate this effect independently of the chosen signal, plotting a square-box frequency filter $F(f)$ (solid line) and the FT of the function $I_{F}$ using the same filter in Fig. 10. It can be seen from this figure that the edges of the square-box frequency window have been smoothed in a symmetric manner and similarly to a taper. Thus, the filter can be understood as a frequency taper. In any case, this can be a welcome process in order to lower Gibbs effect when going back to the time domain.

Figs. 11 and 12 show this filtering on, respectively, an IFT of a cosine and on an IFT of a chirp. In each figure, the top plots represent the filtered FT of the signal whereas the bottom plots represent the filtered time signal. The smoothing effect of $I_{F}$ is clearly seen on these plots.

\section{Example of Time-Frequency Filtering}

The purpose of this part is to illustrate the side effects that occur when filtering using the ST and its inverses on some more 


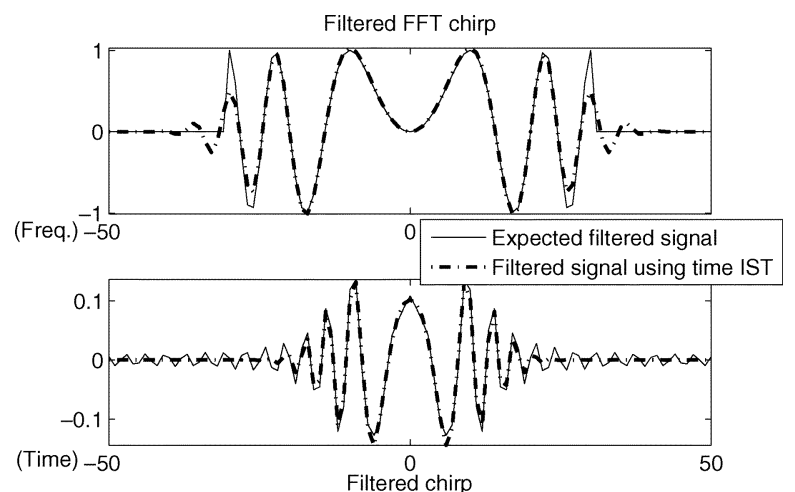

Fig. 12. Filtering of an IFT of a chirp: expected result (solid line) and result through time IST (dashed-dotted line). The top plots represent the filtered signal in the frequency domain. The bottom plots represent the filtered signal in the time domain.

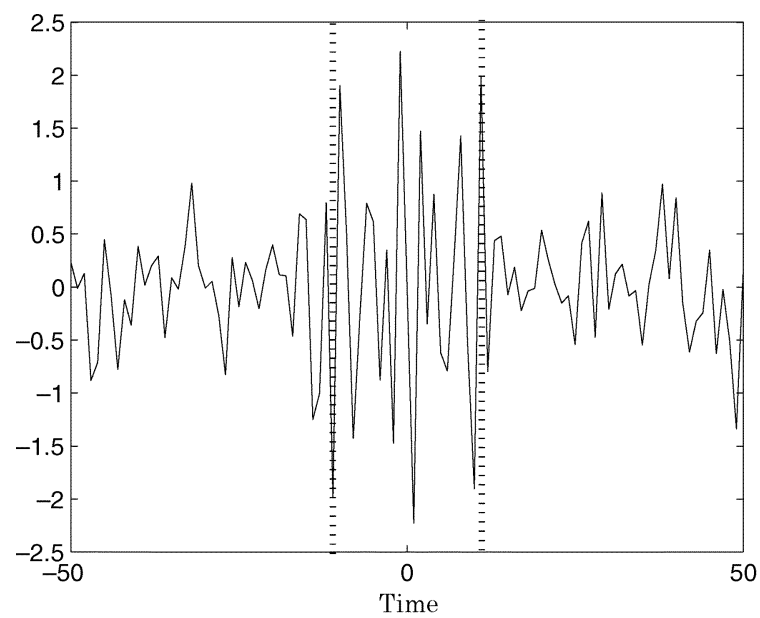

(a)

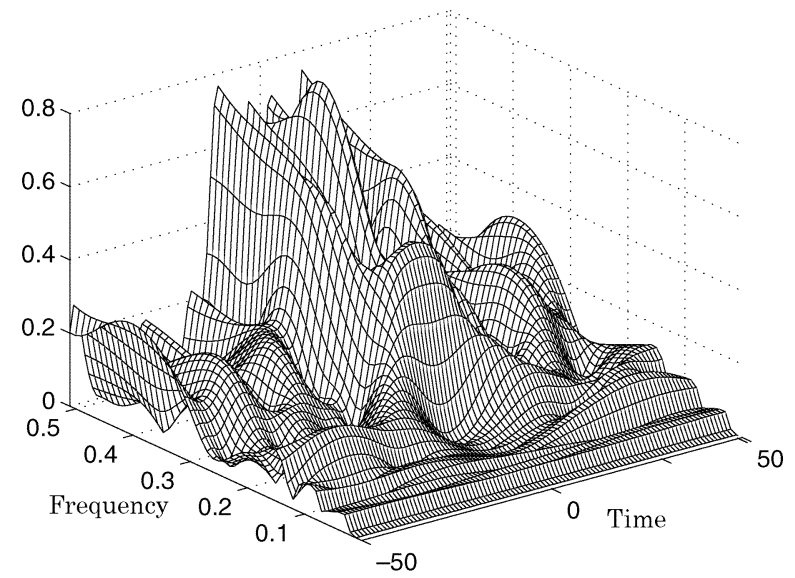

(b)

Fig. 13. Original signal and its time ST. (a) Original signal: the central part between the vertical lines is a sum of three sines. The side parts are random noise. (b) ST of the original signal.

realistic case. The chosen signal is a sum of three sines of frequencies $2.3,3.5$, and $4.3 \mathrm{~Hz}$. We just kept the central part (between $-11 \mathrm{~s}$ and $11 \mathrm{~s}$ ), concatenating some noise on the side parts. The original signal and its ST are plotted in Figs. 13, with the vertical lines representing the limits of the sum of sines. We then apply the square time-frequency weighting function of

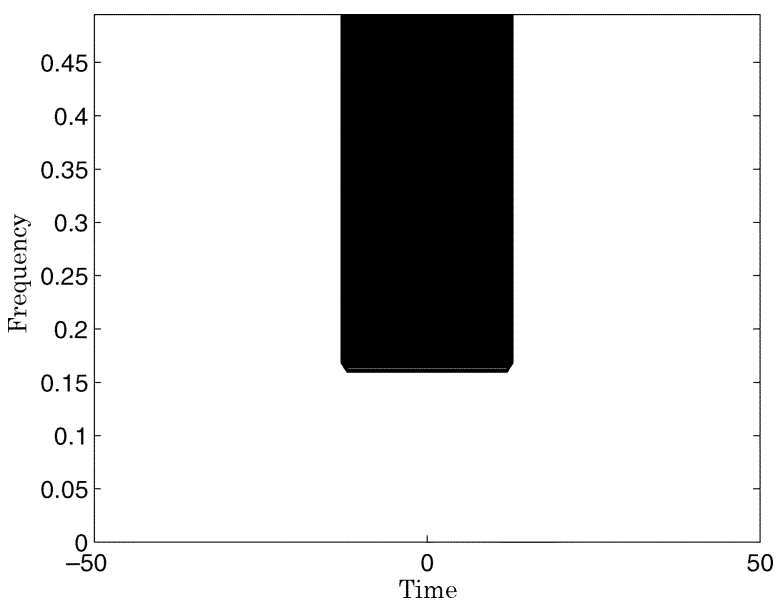

Fig. 14. Mask that will be applied on the original signal [Fig. 13(b)].

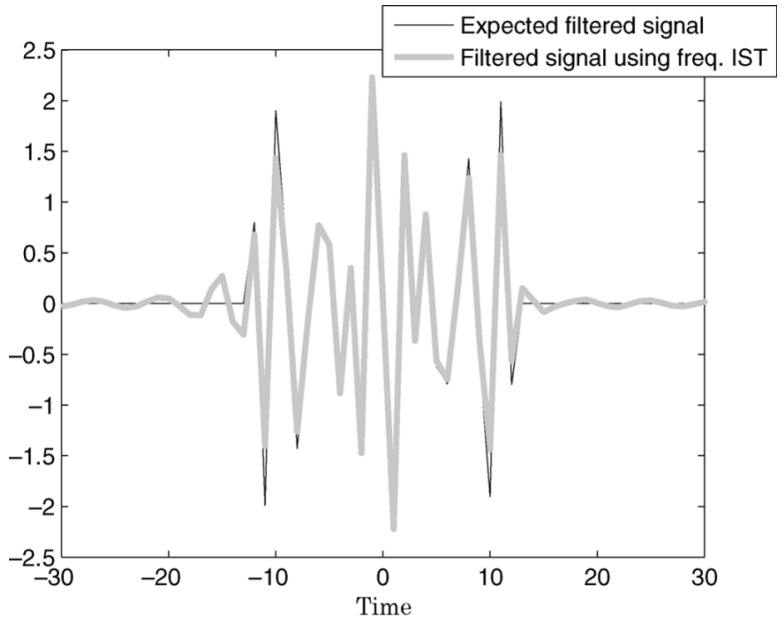

(a)

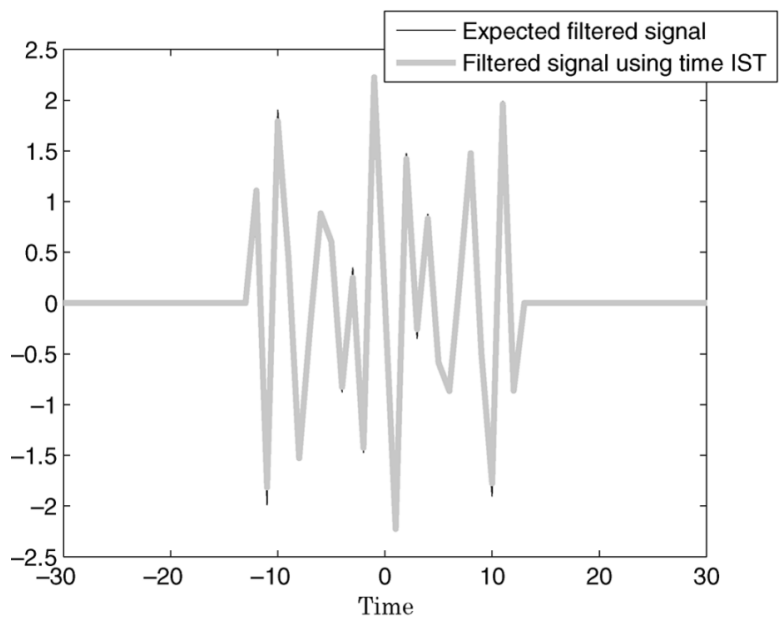

(b)

Fig. 15. Time-frequency filtering a noisy sum of sines [Fig. 13(a)], using the frequency IST on the top plot and the time IST on the bottom plot. (a) Filtering using the frequency IST; (b) filtering using the time IST.

Fig. 14 on this signal. We thus expect to obtain only the central part of the signal, i.e., the sum of sines. The results are presented in Figs. 15 and show the different artifacts that occur using the frequency IST [Fig. 15(a)] and the time IST [Fig. 15(b)]. 
This example is a good illustration of what was pointed out in Section III-B, namely that the time localization of the phasor has been lost using the frequency IST because of the averaging performed in frequency. Indeed, this leads to some oscillations outside of the mask window in Fig. 15(a) which do not appear in Fig. 15(b).

For further practical examples of the filtering with the ST and its inverses, the reader can look at [12].

\section{CONCLUSION}

This paper has allowed a better understanding of the $S$-transform and its two existing inverses ([3] and [8]). In the continuous case, we have shown that the frequency inverse is exact and that the time inverse is nearly exact, with the error being negligible for $k \geq 1$ (which is the most usual case), i.e., after applying the ST and any of its inverses on a signal, we are led back to the original signal. However, in the finite discrete case, we show that one should be careful on how to compute the ST. We also explain why none of the inverses are exact in the finite discrete domain and quantify the different artifacts that occur when discretizing the two inverses. Moreover, we emphasize another drawback of the frequency IST: it obliges the calculation of as many frequency slots as time slots, which can be a major inconvenience when dealing with big data sets such as in geophysic applications. For the discrete-time IST, the level of approximation depends on the number of points and on the tunable scaling factor, $k$. For reasonable values, the discretizes version of the time IST has nearly no side effect.

The last part of this paper gives insights on the effect of these approximations when the ST of a signal is filtered and then back-transformed. In order to obtain a better understanding, we specifically studied the case of time filters and of frequency filters. We show that the use of the frequency ST-IST combination for time-dependent filtering leads to some time localization problem. On the other hand, the use of the time ST-IST combination for frequency-dependent filtering leads to some (sometimes desirable) smoothing effects.

The inverses differ due to their different strategies so care should be taken in the application of them, the best one depending on the application. The time approach may often be the better choice if one is interested in time-domain postprocessing or interpretations. Conversely, the frequency inverse transform can be of advantage whenever frequency separation is more important than time separation.

\section{REFERENCES}

[1] D. Gabor, "Theory of communication," J. Inst. Elect. Eng., vol. 93, pp. 429-457, 1946.

[2] S. Mallat, A Wavelet Tour of Signal Processing, U. London, Ed. New York: Academic, 1998.

[3] R. G. Stockwell, L. Mansinha, and R. P. Lowe, "Localization of the complex spectrum: The $S$ transform," IEEE Trans. Signal Process., vol. 44, no. 4, pp. 998-1001, Apr. 1996.

[4] S. Assous, A. Humeau, M. Tartas, P. Abraham, and J. L'Huillier, "S-transform applied to laser Doppler flowmetry reactive hyperemia signals," IEEE Trans. Biomed. Eng., vol. 53, no. 6, pp. 1032-1037, Jun. 2006.

[5] S. Andino, R. Menendez, C. Lantz, O. Blank, C. Michel, and T. Landis, "Non-stationary distributed source approximation: An alternative to improve localization procedures," Human Brain Mapping, vol. 14, pp. 81-95, 2001.

[6] M. Schimmel, J. Gallart, and C. Simon, "An alternative inverse $S$-transform for filters with time-frequency localization," in Proc. 4th IEEE Symp. Image Signal Processing Analysis (ISPA), Barcelona, Spain, 2005, pp. 424-429.

[7] C. Pinnegar and L. Mansinha, "The $S$-transform with window of abritrary and varying shape," Geophysics, vol. 68, no. 1, pp. 381-385, 2003.

[8] M. Schimmel and J. Gallart, "The inverse S-transform in filters with time-frequency localization," IEEE Trans. Signal Process., vol. 53, no. 11, pp. 4417-4422, Nov. 2005.

[9] I. S. Gradshteyn and I. M. Ryzhik, Table of Integrals, Series, and Products, 6th ed. New York: Academic Press, 2000.

[10] J. Spanier and K. B. Oldham, An Atlas of Functions. Bristol, PA: Hemisphere, 1987.

[11] C. R. Pinnegar, "Comments on 'The inverse s-transform in filters with time-fequency localization',' IEEE Trans. Signal Process., vol. 55, no. 10, pp. 5117-5120, Oct. 2007.

[12] M. Schimmel and J. Gallart, "Frequency-dependent phase coherence for noise suppression in seismic array data," J. Geophys. Res., vol. 112, no. B4, 2006, B04303.

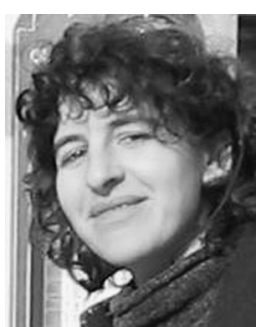

in mobile localization.

She is now at the Technology Marine Unit from the Spanish National Council, Barcelona, Spain, and her main interests are in design of filters for large nonstationary datasets, seismic signal detection and identification, and multiresolution methods.

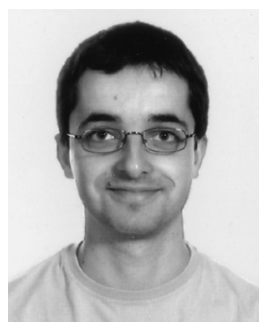

Sergi Ventosa was born in Vilafranca del Penedès, Spain, in 1976. He received the B.S. and M.Sc. degrees in electronics and telecommunication engineering from the Technical University of Catalonia, Barcelona, Spain, in 1999 and 2002. He is currently working toward the Ph.D. degree in signal processing applied to geophysics at the Marine Technology Unit of the Spanish Research Council, Barcelona, Spain. His main interests are nonstationary signal analysis, multidimensional signal processing and pattern recognition techniques.

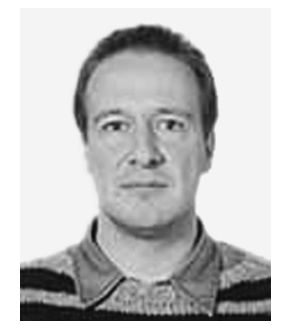

Martin Schimmel received the degree in geophysics from the University of Karlsruhe, Karlsruhe, Germany, in 1992 and the Ph.D. degree from the University Utrecht, Utrecht, The Netherlands, in 1997.

From 1997 to 2001, he was a Postdoctoral Researcher at the Department of Geophysics, IAG, University of Sao Paulo, Brazil. Since 2001, he has been contracted as a Researcher at the Institute of Earth Sciences Jaume Almera of the High Spanish Council for Scientific Research (CSIC), Barcelona, Spain. His current research areas are seismic signal detection and identification, seismic wave propagation, and seismic tomography and migration. 


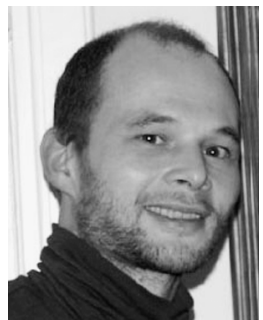

Alexander Heldring was born in The Netherlands in 1966. He received the Master's degree in engineering from the faculty of Applied Physics of the Polytechnic University of Delft, The Netherlands, and the Ph.D. degree from the Polytechnic University of Catalunya, Spain.

After completing his Master's degree, he spent four years in the Sensors, Radar Technologies and Cyber-Security Unit (SERAC) Group at the Joint Research Centre of the European Union in Ispra, Italy. Since 2002, he has been working at the Signal and Processing and Communications Group of the Polytechnic University of Catalunya, Spain. His main interests include antenna modelization and numerical simulation of electromagnetic phenomena.

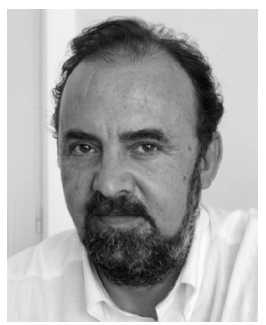

Juan Jo Dañobeitia was born in Santa Cruz Tenerife, Spain, in 1955. He received the M.Sc. degree from the Madrid Complutense University, Spain, and the Ph.D. degree from the Vening Meisnez Laboratory, University of Utrecht, Holland, both in physics.

$\mathrm{He}$ has been an Assistant Professor at the University of Barcelona, Spain, from 1988 to 1990 and the Politechnical University of Catalunya, Spain, from 1992 to 1994 . Since 1992, he has been a Researcher in the Spanish National Council, Barcelona, Spain. He was Director of the Department of Geophysics from 1997 to 2000. Since 2001, he has been Director of the Marine Technology Unit. $\mathrm{He}$ is author or coauthor of over 70 referred publications. His main research interests are in geophysical modeling, seismology, marine technology, continental margin process, and deep oceanic structure.

Dr. Dañobeitia has organized international symposia, and he has been involved in over 30 European or national projects.

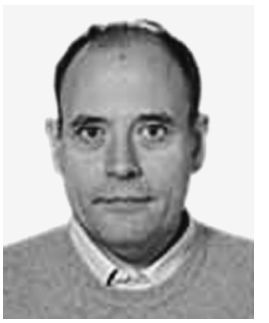

Josep Gallart was born in Barcelona, Spain, in 1954 He received the Physics degree from the University of Barcelona, Spain, in 1976, the Ph.D. degree in earth sciences at the University Pierre et Marie Curie, Paris, France, in 1980, and the Ph.D. degree in geophysics from the University of Barcelona, Spain, in 1981.

He worked at the Institut de Physique du Globe de Paris. In 1985, he joined the University of Barcelona as Professor and moved in 1988 to a research position at the Institute of Earth Sciences-CSIC, Spain, where he has held a Research Professorship since 1999.

He has accomplished internationally recognized research in earth sciences, focused on studies on the Earth structure and dynamics, based on techniques of seismology, seismic imaging, and tectonophysics. He has led and coordinated over 20 national and international projects in different tectonic environments, studying the structure and dynamics of the lithosphere, continental margins, subduction zones, volcanic systems, etc. He has supervised ten Ph.D. dissertations

Dr. Gallart has been a member of the ORFEUS Board of Directors since 1989 and a Spanish representative in the Commission of Controlled Source Seismology (CCSS) of the International Association of Seismology and Physics of the Earth Interior (IASPEI) since 1996. He has been a member and Spanish representative in the European initiatives EuroArray and Topo-Europe since 2005. He has been Vice-Director of the Institute of Earth Sciences and Head of the Geophysics Department of that Institute. He has been a member of the Earth Sciences Evaluation Panel of the Spanish Agency (ANEP) since 2004.

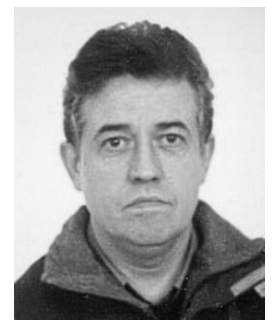

Antoni Mànuel was born in Barcelona, Spain, in 1954. He received the telecommunication engineering degree and the Ph.D. degree in telecommunication engineering from the Technical University of Catalonia, Spain, in 1980 and 1996, respectively.

Since 1988, he has been an Associate Professor in the Department of Electronic Engineering at the Technical University of Catalonia, where he has been Director of the research group Remote Acquisition Systems and Data Processing (SARTI) since March 2001. He is also the Coordinator of the Tecnoterra associated unit of the Scientific Research Council through the Jaume Almera Earth Sciences Institute and Marine Science Institute. His current research interests are in applications of automatic measurement systems based on the concept of virtual instrumentation and oceanic environment. He is currently involved in more than ten projects with the industry and seven funded public research projects. 